\title{
Improving the Performance of Distributed Virtual Environment Systems
}

P. Morillo, J. M. Orduña, M. Fernández, and J. Duato, Member, IEEE

This paper is supported by the Spanish MCYT under Grant TIC2003-08154-C06-04

P. Morillo, J. M. Orduña and M. Fernández are with Departamento de Informática, Universidad de Valencia (SPAIN).

J. Duato is with DISCA, Universidad Politécnica de Valencia (SPAIN). 


\begin{abstract}
Last years have witnessed a dramatic growth in the number as well as in the variety of Distributed Virtual Environment systems. These systems allow multiple users, working on different client computers that are interconnected through different networks, to interact in a shared virtual world. One of the key issues in the design of scalable and cost-effective DVE systems is the partitioning problem. This problem consists of efficiently assigning the existing clients to the servers in the system, and some techniques have been already proposed for solving it.

This paper experimentally analyzes the correlation of the quality function proposed in the literature for solving the partitioning problem with the performance of DVE systems. Since the results show an absence of correlation, we also propose the experimental characterization of DVE systems. The results show that the reason for that absence of correlation is the non-linear behavior of DVE systems with regard to the number of clients in the system. DVE systems reach saturation when any of the servers reaches $100 \%$ of CPU utilization. The system performance greatly decreases if this limit is exceeded in any server. Also, as a direct application of these results we present a partitioning method that is targeted to keep all the servers in the system below a certain threshold value of CPU utilization, regardless of the amount of network traffic. Evaluation results show that the proposed partitioning method can improve DVE system performance, regardless of both the movement pattern of clients and the initial distribution of clients in the virtual world.
\end{abstract}

\title{
Index Terms
}

Distributed Applications, Distributed/network graphics

\section{INTRODUCTION}

Professional high performance graphic cards currently offer a very good frame-rate for rendering complex 3D scenes in real time. Fast Internet connections have also become worldwide available at a relatively low cost. These two factors have made possible the current growth of Distributed Virtual Environment (DVE) Systems. These systems allow multiple users, working on different client computers that are interconnected through different networks (and even through the Internet) to interact in a shared virtual world. This is achieved by rendering images of the environment as the user would perceive them if he was located in the virtual environment. Each user is represented in the shared virtual environment by an entity called avatar, whose state is controlled by the user through the client computer. Since DVE systems support visual interactions between multiple avatars, each client computer needs to be reported about any 
positional change of those avatars in the neighborhood of the avatar controlled by that client computer. DVE systems are currently used in many different applications [39], such as civil and military distributed training [31], collaborative design [37] and e-learning [5]. Commercial multi-player game environments are also considered as DVE systems [2], [23], [42], since these systems address the same issues and use the same simulation techniques that DVE systems do [38].

DVE systems can be based on different platforms. Different factors make DVE systems to be inherently heterogeneous:

- Each client computer controlling an avatar may be completely different. A very different performance can be found when comparing not only different processors, but also other computer resources such as memory size and speed, etc. Each client computer can have a different combination of these and other resources.

- Different networks can be found in a single DVE system. Since each network can have any existing network technology, different network technologies can be simultaneously found in a single DVE system.

- Depending on the application, avatars can show different communication rates. For example, the communication rate of avatars in a collaborative 3-D environment may greatly differ from the communication rate of avatars in a 3-D virtual military battle.

As a result, the establishment of a general methodology for the design of an effi cient DVE system is a complex task. Nevertheless, some aspects of DVE systems have been studied:

- Data Model: This concept describes some conceivable ways of distributing persistent or semi-persistent data in a DVE [28]. Data can be managed using a replicated, shared, or a distributed strategy.

- Communication Model: The way that avatars communicate with each other has an effect on the system performance. Different communication schemes, such as broadcast, peer-topeer [21] or unicast determine different network traffi c patterns for exchanging information between avatars.

- View Consistency: This problem has been already defi ned in other computer science fi elds such as database management [6]. View consistency consists of ensuring that all avatars sharing a virtual space have the same vision on all the objects in that virtual space. 
- Network Traffic Reduction: Keeping a low number of messages allows DVE systems to effi ciently scale with the number of avatars in the system. Traditionally, techniques like dead-reckoning offered some level of independence to avatars [14], [18]. Also, broadcast or multicast solutions allow to decrease the number of messages required for keeping a consistent state of the system [11], [22], [42].

One of the key issues in the design of a scalable and effi cient DVE system is the partitioning problem [13]. It consists of effi ciently assigning the workload (avatars) among the servers in the system. The partitioning problem determines the overall performance of the DVE system, since it has an effect not only on the workload that each server in the system is assigned to, but also on the inter-server communications (and therefore on the network traffic). Some methods for solving the partitioning problem have been already proposed [13], [26], [27], [39]. However, there are still some features in the proposed methods that can be improved. For example, instead of using the ad-hoc search method proposed in [27], several heuristic search methods can be used for solving this problem [32], [33]. Nevertheless, the main task that should still be done is to correlate the quality function used in the search method with DVE system performance. Otherwise, the proposed method could provide ineffi cient solutions for the partitioning problem.

This paper experimentally studies if the quality function used in the literature for solving the partitioning problem actually measures in an effective way the different performance levels that different partitions should produce in the same DVE system. Since the results show a total absence of correlation, this paper also studies the experimental characterization of DVE systems. This characterization study measures the impact of different parameters of the quality function on the performance of DVE systems [34]. The results show that the behavior of DVE systems is non-linear with the number of avatars in the system, regardless of the value of the quality function. Also, the results show that the performance of DVE systems greatly decreases when any of the servers reaches $100 \%$ of CPU utilization. Therefore, as a direct application of these results we propose a partitioning method that is targeted to keep all the servers in the system below a certain threshold of CPU utilization, regardless of the amount of network traffi c. Evaluation results show that the proposed partitioning method can improve DVE system performance, regardless of both the movement pattern of avatars and also the initial distribution of avatars in the virtual world.

The rest of the paper is organized as follows: Section II describes the partitioning problem 
and the existing proposals for solving it. Section III details the proposed characterization setup, which allows to experimentally study the behavior of DVE systems. Next, Section IV presents the correlation and characterization results. Section V describes a new partitioning method that takes into account the characterization results shown in the previous section. Finally, Section VI presents some concluding remarks and the future work to be done.

\section{The Partitioning Problem in DVE Systems}

Architectures based on networked servers are becoming a de-facto standard for DVE systems [17], [24], [28], [39], [40]. In these architectures, the control of the simulation relies on several interconnected servers. Client computers are attached to one of the servers in the system. When a client computer modifi es the state (usually the position) of an avatar, it also sends an updating message to its server, which in turn must propagate this message to other servers and clients. Servers must render different 3-D models, perform positional updates of avatars and transfer control information among different clients. Thus, each new avatar represents an increase in both the computational requirements of the application and in the amount of network traffi c. When the number of connected clients increases, the number of updating messages must be limited in order to avoid a message outburst. In this sense, different approaches have been proposed in order to limit the number of neighboring avatars that a given avatar must communicate with. Concepts like areas of influence (AOI) [39], [42], locales [3] or auras [15] defi ne a neighborhood area for avatars, in such a way that a given avatar must notify its movements (by sending an updating message) only to those avatars located in its neighborhood. Thus, the AOI of a given avatar determines the amount of network traffi c generated by that avatar. Other approaches use three tiered architectures [1], [22], data fi ltering [41] or distributed cache management [8] in order to minimize the impact of network traffi c on the performance of the DVE system.

Depending on their origin and destination avatars (client computers), messages in a DVE system can be intra-server or inter-server messages. In order to design a scalable DVE system, the number of inter-server messages should be minimized. Since intra-server messages only require a single server, the more intra-server messages there are, the less resources the application will require to exchange the same number of messages. Fig. 1 shows an example of a DVE system with a multi-server architecture, composed of three servers. It also shows an example of both intra-server and inter-sever communication. 


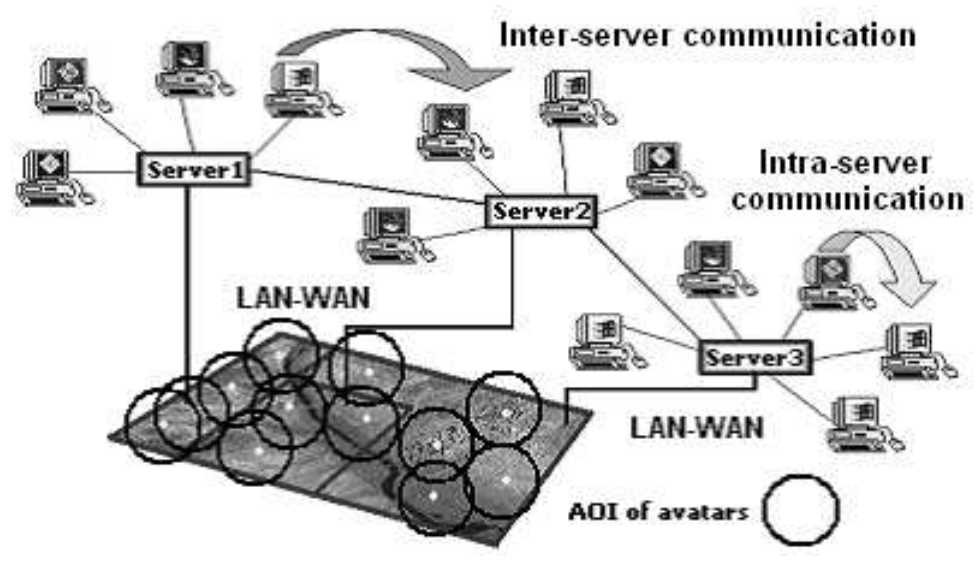

Fig. 1. An example of multi-server architecture for a DVE system

Lui and Chan have shown the key role of fi nding a good assignment of avatars (clients) to servers in order to ensure both a good frame rate and a minimum network traffi c in DVE systems [27]. They propose a quality function for evaluating each assignment of clients to servers (partition), denoted as $C_{p}$. This quality function takes into account two parameters. One of them measures how balanced the computing workload is in that partition, which is denoted as $C_{p}^{W}$. In order to minimize this parameter, the computing workload should be proportionally distributed among all the servers in the DVE system, according to the computing resources of each server. The other parameter of the quality function measures the overall inter-server communication requirements, which is denoted as $C_{p}^{L}$. In order to minimize this parameter, avatars sharing the same AOI should be assigned to the same server. Quality function $C_{p}$ is defi ned as

$$
C_{p}=W_{1} \cdot C_{p}^{W}+W_{2} \cdot C_{p}^{L}
$$

where $W_{1}+W_{2}=1 . W_{1}$ and $W_{2}$ are two coefficients that weight the relative importance of the computational and communication workload, respectively. These coeffi cients should be tuned according to the specific features of each DVE system. Thus, if the servers in the DVE system are connected through low performance networks (i.e Internet), then the quotient $W_{1} / W_{2}$ must be close to zero. On the contrary, if the servers are interconnected using high performance networks, the quotient $W_{1} / W_{2}$ must be close to one. Using the quality function $C_{p}$ (and assuming $W_{1}=W_{2}=0.5$ ), Lui and Chan propose a partitioning algorithm that must be periodically 
executed [25], [26], in order to adapt the partition to the current state of the DVE system (avatars can join or leave the DVE system at any time, and they can also move everywhere within the simulated virtual world). Lui and Chan have further suggested a testing platform for the performance evaluation of DVE systems, as well as a parallelization of the partitioning algorithm [27].

Other approaches for solving the partitioning problem have been also proposed. One of them [29] groups avatars following regular distributions. In order to ensure good performance, this approach generates a number of regular distributions equal to the number of servers in the DVE system. However, this proposal does not obtain good performance when avatars are located following a non-uniform distribution.

A different approach rejects dynamic concepts associated to avatars like AOI, aura or locale [40]. This proposal divides the 3D virtual scene into a regular grid. A multicast group is created for each grid cell, in such a way that avatars sharing a cell also share multicast packets and are assigned to the same server. Although this approach provides a fast way of solving the partitioning problem, the performance of the static partitioning is quite low when avatars are located following a clustered distribution. In this case, the servers controlling the areas where clusters are located are overloaded, increasing the overall cost of the quality function. A similar idea has been also suggested using unicast communications [7].

The partitioning method proposed by Lui and Chan currently provides the best results for DVE systems. However, the correlation of the quality function $C_{p}$ proposed by Lui and Chan [27] with DVE system performance has not been studied yet, and the parameters $W_{1}$ and $W_{2}$ should still been tuned. In this sense, the characterization of DVE systems is essential to design partitioning strategies that actually improve the performance of DVE systems.

\section{Characterization Setup}

We propose the characterization of generic DVE systems by simulation. The evaluation methodology used is based on the main standards for modeling collaborative virtual environments, such as FIPA [12], DIS [10] and HLA [20]. We have developed a simulation tool (written in C++), which models the behavior of a generic DVE system composed of several interconnected servers. Concretely, we have implemented a set of multi-threaded servers (each server on a different, dedicated computer). Each thread in a server uses blocking sockets for communicating with a 
client computer. Each client controls the behavior of a single avatar, and it is also implemented as a multi-threaded application. One of the threads manages the communication with the server assigned to that client, while another thread manages user information (current position, network latency, etc.).

Our simulator models a DVE system composed of $S$ interconnected servers and $n$ avatars. Following the approach specifi ed by FIPA and HLA standards, one of the servers acts as the main server (called Agent Name Service in [12] or Federation Manager in [20]) and it manages the whole system. Avatars can join the simulation through this main server, which uses a partitioning fi le for assigning each new avatar to one of the servers in the system. In each simulation, all avatars sharing the same AOI must communicate among them (by sending updating messages) in order to notify their position in the 3-D virtual world. The message structure used for notifying avatar movements is the Avatar Data Unit (ADU) specifi ed by DIS [10].

A simulation consists of each avatar performing 100 movements, at a rate of one movement every 2 seconds. Each time an avatar performs a movement, the client computer controlling that avatar notifi es that movement to its server by sending a message with a timestamp. The server must then notify the movement to all the client computers controlling the avatars in the same AOI of the sender avatar. When that notifi cation arrives at the destination client computers, they return an ACK message to the server, which in turn propagates these ACK messages to the sender client computer.

When an ACK message arrives, the sender client computer can measure the round-trip delay for each message sent. We have denoted such round-trip delay (measured in real-time) as the system response. Since destination client computers send the ACK message back to the client computer controlling the sender avatar, the same clock is used for both the initial and final timestamp. In this way, clock skewing is avoided when computing system latency. When a simulation ends, each avatar (the client computer controlling each avatar) has computed the average system response for communicating with the avatars in its AOI. At this point, all avatars send their average system responses to their respective servers, which in turn compute their own average system response. Finally, starting from the average value provided by each server, the main server computes the average system response for the entire system and for that simulation, denoted as the average system response (ASR). An effi cient DVE system must keep ASR value as low as possible, regardless of the number of avatars in the system. On the other hand, system 
throughput consists of the maximum number of avatars that the system can support without greatly increasing the ASR. Since the ASR (average system latency) shows how fast users perceive the system, we have considered the ASR as the main performance measure in order to characterize the behavior of DVE systems.

\section{Simulation AND CORRElation Results}

In this section, we present the results provided by the simulation model described in the previous section. First, we present the characterization of the workload generated by avatars in a DVE system. Next, we present the correlation of the quality function $C_{p}$ with the DVE system performance. Finally, we present the characterization of DVE systems [34].

We have performed about 2,000 experiments on a wide variety of DVE systems with a multiserver architecture, ranging from small virtual worlds (3 servers and 180 avatars) to large virtual worlds (900 avatars and 6 servers). Since we have obtained very similar results in all of them, for the sake of shortness we present only the results for a small DVE confi guration.

Due to characterization purposes, in each simulation each client has only performed a fi xed number of movements at a fi xed rate. We have also assumed that no changes are produced in any static element of the virtual world and the AOI of avatars has the same size during the whole simulation. Although these assumptions are not realistic, they only have an effect on the workload produced by avatars, but they do not have any effect on the behavior of DVE servers.

The hardware platform used for simulating both clients and servers in the DVE system have been PC's with processor Pentium IV at $1.7 \mathrm{GHz}$, with 256 Mbytes of RAM and with NVidia MX-400 graphic cards. Each server has been implemented in a single PC, while up to 50 clients have been allocated in the same PC. We have used a $10 \mathrm{Mbps}$ Ethernet as the interconnection network. The simulation tool has been executed on a Windows 2000 Professional operating system.

\section{A. Characterization of Avatars}

The first step in the characterization of DVE systems is to estimate the workload generated by avatars. It can be seen that, when avatars move faster, then the CPU utilization in DVE servers increases. The same behavior can be observed when avatars are located following uneven 
distributions within the scene. Therefore, we have proposed a model that takes into account both factors in order to estimate the workload generated by avatars [35].

Concretely, we define the presence factor $P_{f}(i)$ as the number of avatars in whose AOI avatar $i$ appears. This presence factor and the movement rate of avatar $i$ will determine the network traffi $\mathrm{c}$ and the workload generated by avatar $i$ [35]. Effectively, when the avatars that are allocated in a given server change the number of movements per second (movement rate) in the $3 \mathrm{D}$ virtual scene, then there is a variation in the number of messages to be processed by that server. Therefore, when a given avatar moves faster it will require more CPU utilization in order to process its messages. We have measured the percentage increase of CPU utilization due to changes in the movement rate of avatars. A significant example is shown in Fig. 2. The results shown in this figure have been obtained from a DVE system composed of 180 avatars and 3 servers. This fi gure shows on the $\mathrm{X}$-axis the values of movement rate accomplished by all the avatars in the simulation. The Y-axis shows ASR (Y-left) and CPU utilization (Y-right) values for several simulations performed with the same partition. Each point in the plot represents the average value of the ASR and CPU utilization obtained after 30 simulations of the same DVE system. The standard deviation for any of the points shown in the plot was not higher than 30 ms. for the ASR and $4 \%$ for the CPU utilization in any case.

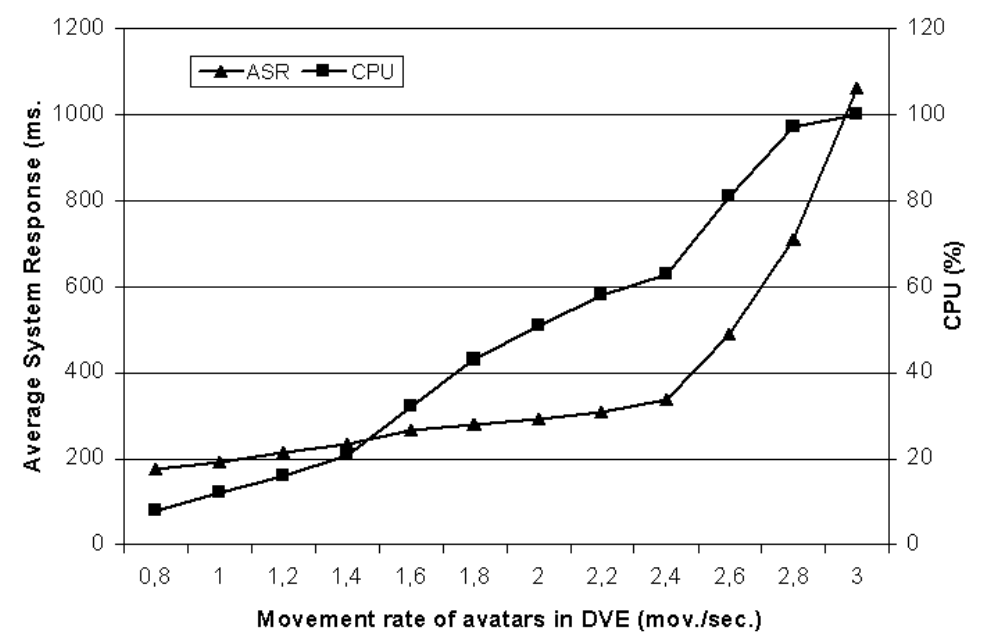

Fig. 2. Variation of CPU utilization and ASR with the movement rate of avatars

Fig. 2 shows that the workload generated by avatars (in terms of both ASR and CPU utilization) 
is related to their movement rate. Therefore, the movement rate of avatars can provide a way of estimating the workload generated by avatars.

Additionally, Fig. 3 shows the behavior of a DVE system when the presence factor of avatars is modifi ed. This fi gure shows on the $\mathrm{X}$-axis the values for the presence factor of all avatars in a DVE system. The Y-axis shows the ASR and the average CPU utilization values for different simulations of the same partition when different values for the presence factor are used.

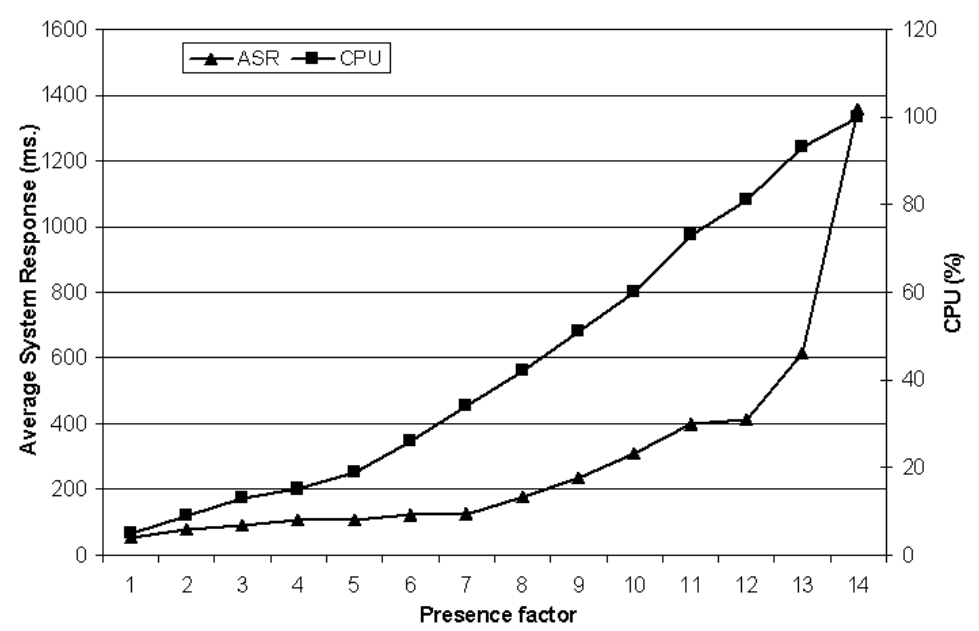

Fig. 3. Variation of CPU utilization and ASR with the presence factor of avatars

Fig. 3 helps to explain why, when avatars come closer in the virtual space, then the workload generated by these avatars increases. The grouping causes an increase of the $P_{f}$ of the avatars, and therefore, an increase in the amount of updating messages sent by each one of them when they move. These new messages mean more CPU utilization for accomplishing the task. Based on these results, we have proposed a method for estimating the workload that avatars generate to a given DVE system [35].

In order to evaluate the performance of a given partitioning method, usually 3 different avatar distributions in the virtual world have been suggested in the literature: uniform, skewed and clustered [27]. The reason for using different distributions is that they generate a different workload. Fig. 4 shows an example of these avatar distributions in a 2-D virtual world. In this fi gure, the virtual world is a square and avatars are represented as black dots.

However, the workload that each avatar represents for the server where it is assigned to can be made independent from the distribution of avatars in the virtual world. Since different avatar 


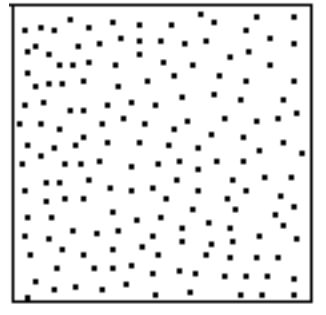

a)

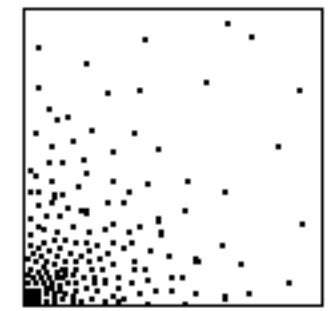

b)

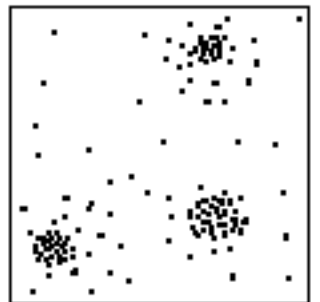

c)

Fig. 4. Distributions of avatars in a 2-D virtual world: a) Uniform b) Skewed c) Clustered

distributions in the virtual world only differ in the workload generated by avatars, only one of the distributions is necessary for evaluation purposes. The effect of testing the partitioning method with different avatar distributions can be obtained by changing the presence factor and the movement rate of avatars. Therefore, for the purpose of characterizing DVE systems we have considered only a uniform distribution of avatars, with the same AOI and the same movement rate for all avatars in the system.

\section{B. DVE Systems Characterization}

Taking into account the characterization of both the workload generated by avatars shown above, in this subsection we analyze the correlation of quality function $C_{p}$ and the DVE systems characterization.

Fig. 5 shows the performance results for a small DVE system composed of 3 servers and 180 avatars when different partitions (showing different values of the quality function $C_{p}$ ) are simulated. This figure shows on the $\mathrm{X}$-axis the values of the quality function $C_{p}$ obtained for different partitions (assignments of avatars to the servers in the system). The Y-axis shows ASR values for the simulations performed with these partitions. Each point in the plot represents the average value of ASR obtained after 30 simulations of the same DVE system under the same workload. The standard deviation for any of the points shown in the plot was not higher than $25 \mathrm{~ms}$. in any case.

Fig. 5 clearly shows that $C_{p}$ does not correlate with DVE system performance. The ASR values hardly change as $C_{p}$ values are greatly increased. These results do not show a linear correspondence between the values of quality function $C_{p}$ and the ASR values. Therefore, a 


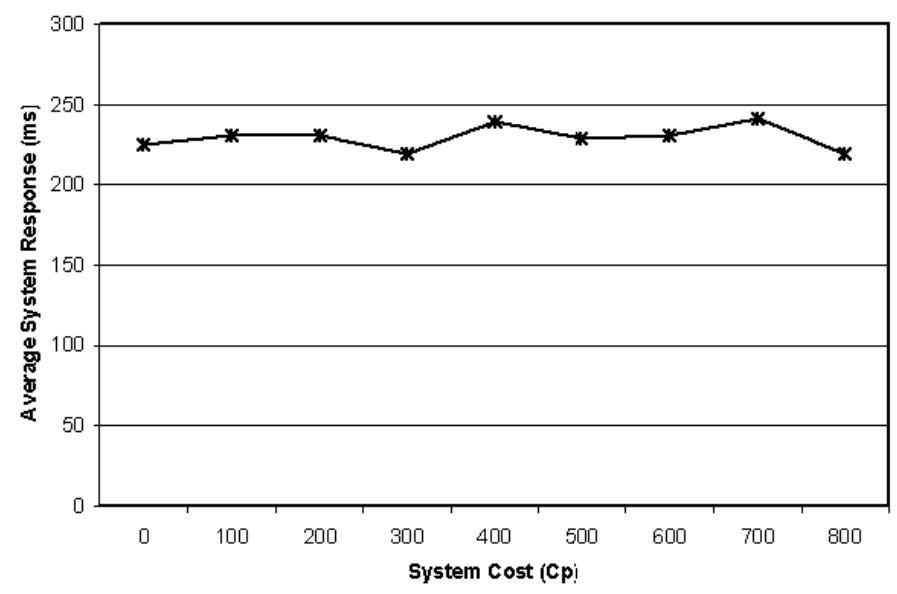

Fig. 5. Correlation of quality function $C_{p}$ with average system response

characterization study is needed in order to capture the behavior of DVE systems.

The first goal of our characterization study was to find out the reason for the absence of correlation between $C_{p}$ and the system performance. In order to achieve this goal, we studied the behavior of DVE systems as the number of avatars in the system increases. Fig. 6 shows the ASR values obtained for different DVE confi gurations, composed of 3, 4 and 5 servers, as the number of avatars in the system increases. In this fi gure, the $\mathrm{X}$-axis shows the total number of avatars in the DVE system. The value of $C_{p}$ associated to the simulated partitions was zero in all cases (perfect workload balancing and absence of inter-server messages, that is, $C_{p}^{L}$ and $C_{p}^{W}$ equal to zero). ASR values seem to be invariant with regard to the number of avatars in the system (ASR plot has the shape of a flat line) until a saturation point is reached. From that saturation point, ASR values greatly increase as new avatars are added to the system. These results clearly show that the behavior of a DVE system is non-linear in relation to the number of avatars in the system. However, $C_{p}$ function is defi ned (1) as a linear function of both workload balancing and inter-server communications. Therefore, this non-linear behavior can explain the absence of correlation shown in Fig. 5.

In order to determine the reason for the non-linear behavior of DVE systems, Table I shows the CPU utilization and the average system responses corresponding to simulation results (DVE confi guration of 3 servers) shown in Fig. 6. The upper part of this table shows the percentage of CPU utilization for each server in the system, as well as the ASR value (in milliseconds) 


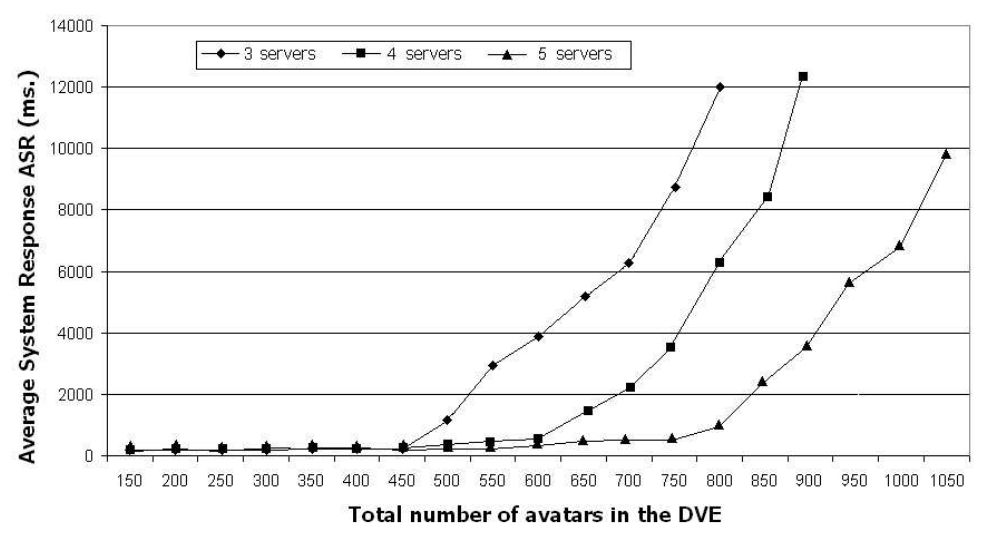

Fig. 6. Variation of average system responses with the number of avatars in the system

obtained for each simulation. The left column shows the total number of avatars in the system for each simulation, and how these avatars are distributed among the three servers. The lower part of this table shows the average system responses (in milliseconds) obtained for each server (SR-Sx) corresponding to the same simulations. Also, the global ASR value is shown again on the right column. This table shows that the DVE system reaches the saturation point when some of the servers in the system reach a $100 \%$ CPU utilization (for 800 avatars, the CPU utilization in servers 1,4 and 5 reach $100 \%$, while for this simulation the ASR increases greatly). All the SR-Sx values increase as the respective CPU utilization does. Also, there are not signifi cant differences between the three SR-Sx values while the CPU utilization remains under $100 \%$. However, when the CPU utilization surpasses $99 \%$ in some servers, then the system response for that server increases greatly, also increasing the global ASR accordingly. Therefore, we can conclude that the non-linear behavior of DVE systems shown in Fig. 6 is due to the $100 \%$ limit in any CPU utilization. Since the quality function $C_{p}$ does not take into account the CPU utilization in order to measure the quality of a partition, it cannot take into account the non-linear behavior of DVE systems as the number of avatar increases either. For example, a given partition can be composed of too many avatars for a given server (thus providing a poor system performance) and, at the same time, it can be a well-balanced partition, resulting in a very low $C_{p}$ value.

These results indicate that, although a partition can provide perfect workload balancing and minimum inter-server communication requirements (thus providing an optimum $C_{p}$ value, as the partitions in Fig. 6 do), ASR will be very poor if the number of avatars assigned to some 
TABLE I

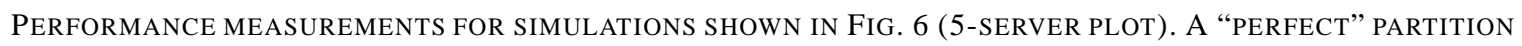
$\left(C_{p}=C_{p}^{W}=C_{p}^{L}=0\right)$ HAS BEEN USED FOR THAT PLOT

\begin{tabular}{|c|c|c|c|c|c|c|}
\hline \multicolumn{7}{|c|}{ Utilization (\%) } \\
\hline Number of avatars & CPU1 & CPU2 & CPU3 & CPU4 & CPU5 & ASR \\
\hline $\mathbf{6 0 0}(120,120,120,120,120)$ & 51 & 54 & 52 & 53 & 51 & 205.7 \\
\hline $700(140,140,140,140,140)$ & 71 & 69 & 76 & 78 & 74 & 274.0 \\
\hline $800(160,160,160,160,160)$ & 100 & 98 & 95 & 100 & 100 & 1001.4 \\
\hline $900(180,180,180,180,180)$ & 100 & 100 & 100 & 100 & 100 & 3068.3 \\
\hline $1000(200,200,200,200,200)$ & 100 & 100 & 100 & 100 & 100 & 4172.2 \\
\hline \multicolumn{7}{|c|}{ System Responses (ms) } \\
\hline Number of avatars & SR-S1 & SR-S2 & SR-S3 & SR-S4 & SR-S5 & ASR \\
\hline $\mathbf{6 0 0}(120,120,120,120,120)$ & 206.16 & 199.5 & 204.9 & 227.1 & 190.6 & 205.7 \\
\hline $700(140,140,140,140,140)$ & 284.2 & 260.8 & 265.9 & 287.4 & 271.6 & 274.0 \\
\hline $800(160,160,160,160,160)$ & 998.5 & 965.5 & 897.5 & 1158.5 & 987.1 & 1001.4 \\
\hline $900(180,180,180,180,180)$ & 3158.2 & 3152.2 & 2857.5 & 3215.5 & 2958.2 & 3068.3 \\
\hline $1000(200,200,200,200,200)$ & 4095.1 & 4199.2 & 4201.2 & 4012.0 & 4193.2 & 4172.2 \\
\hline
\end{tabular}

servers requires a CPU utilization ranging from $95 \%-100 \%$ or more. Also, given a DVE system and given a certain number of avatars in the system, the performance of that DVE system will remain practically invariant with regard to $C_{p}$ if none of the servers reaches a CPU utilization close to $100 \%$. This is the case for the simulations whose results are shown in Fig. 5, where only $90 \%$ of the CPU utilization was reached in the worst case.

In order to prove that the correlation results shown in Fig. 5 can be explained by the non-linear behavior of DVE systems shown in Fig. 6, Fig.7 shows the CPU utilization measured in each server for those simulations. Fig.7 shows on the $\mathrm{X}$-axis the values of the quality function $C_{p}$, as Fig. 5 does. However, in this fi gure for each $C_{p}$ value the CPU utilization of each server is shown as a bar diagram. The numbers on the top of each bar diagram indicate the number of avatars assigned to each server. The Y-axis show the percentage of CPU utilization. 


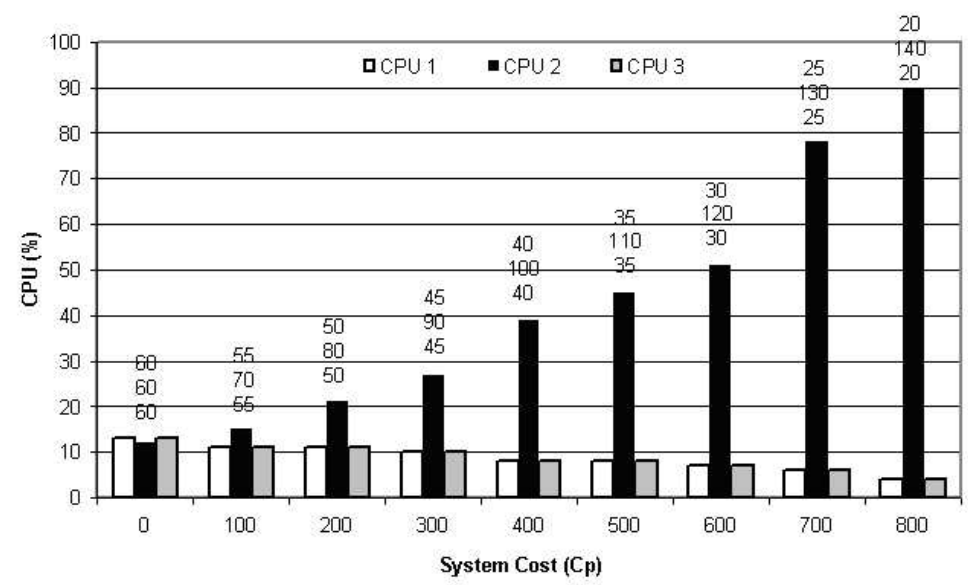

Fig. 7. CPU utilization for the experiments shown in Fig. 5

Fig. 7 reveals that none of the servers in the DVE system reaches $100 \%$ of CPU utilization, even for the worst partition considered. Also, this Figure shows that all the new avatars are assigned to the same server in order to increase the value of the quality function $C_{p}$ in different partitions. In this way, workload is unbalanced and, therefore, $C_{p}$ is increased. When $C_{p}$ reaches a value of 800 (the worst case considered in Figure 5, where server 2 only reaches $90 \%$ of CPU utilization. That is, this DVE system is working on the "flat line" zone of Figure 6 for all the partitions considered. The resulting workload for server 2 generated by any of the partitions does not lead to system saturation. Therefore, all the partitions considered result in a similar ASR value.

The next step when making a methodical characterization study of DVE systems was to study the behavior of DVE systems as the two terms of the sum in (1) vary. Given a DVE system composed of 3 servers, Fig. 6 and Table I illustrate the behavior of the DVE system with the best partitions as possible (the values of $C_{p}^{W}$ and $C_{p}^{L}$ are both equal to zero). Next, we have optimized the $C_{p}^{W}$ value (workload balancing) and we have simulated (under the same workload) both a partition with an optimum (minimized) $C_{p}^{L}$ value (inter-server communication requirements) and also a partition with the worst $C_{p}^{L}$ value as possible. Additionally, for the same DVE system we have changed the partitions in order to obtain the worst $C_{p}^{W}$ value as possible, performing the same simulations with the partitions showing both the best and the worst values of the term $C_{p}^{L}$.

Table II shows the simulation results for the same DVE confi guration whose results are shown 
in Table I. However, this confi guration has been simulated now under partitions with the worst value of $C_{p}^{L}$ as possible (the maximum number of inter-server messages). When comparing Table I and Table II in a line-by-line analysis, we can see that an increase in the number of inter-server messages produces a fi xed (but not signifi cant) increase in ASR values if the system is not saturated (the difference between the ASR values in both tables in the first two lines remains constant, about $48 \mathrm{~ms}$.). The effect of the number of inter-server messages in the ASR values becomes more important as servers enters deep saturation. Also, we can see that for the same number of avatars (rows corresponding to 600 and 700 avatars) Table II shows slightly higher CPU utilization values. However, these differences are not signifi cant. Therefore, if the workload is well balanced, then the number of inter-server messages does not have a signifi cant effect on the system throughput (the number of avatars that a given DVE system can manage without enter saturation), nor on the system latency (ASR).

TABLE II

PERFORMANCE RESUlts FOR A DVE CONFIGURATION OF 5 SERVERS AND A PARTITION WITH MINIMIZED $C_{p}^{W}$ TERM AND MAXIMIZED $C_{p}^{L}$ TERM

\begin{tabular}{|c|c|c|c|c|c|c|}
\hline \multicolumn{7}{|c|}{ Utilization (\%) } \\
\hline Number of avatars & CPU1 & CPU2 & CPU3 & CPU4 & CPU5 & ASR \\
\hline $\mathbf{6 0 0}(120,120,120,120,120)$ & 62 & 59 & 61 & 59 & 60 & 250.2 \\
\hline $700(140,140,140,140,140)$ & 70 & 76 & 84 & 81 & 88 & 322.4 \\
\hline $800(160,160,160,160,160)$ & 99 & 98 & 100 & 100 & 100 & 1121.7 \\
\hline $900(180,180,180,180,180)$ & 100 & 100 & 100 & 100 & 100 & 3375.2 \\
\hline $1000(200,200,200,200,200)$ & 100 & 100 & 100 & 100 & 100 & 4824.4 \\
\hline \multicolumn{7}{|c|}{ System Responses (ms) } \\
\hline Number of avatars & SR-S1 & SR-S2 & SR-S3 & SR-S4 & SR-S5 & ASR \\
\hline $\mathbf{6 0 0}(120,120,120,120,120)$ & 259.5 & 249.7 & 265.4 & 249.8 & 226.7 & 250.2 \\
\hline $700(140,140,140,140,140)$ & 341.9 & 298.8 & 292.5 & 336.1 & 342.6 & 322.4 \\
\hline $800(160,160,160,160,160)$ & 1187.5 & 1085.8 & 1274.4 & 998.9 & 1062.1 & 1121.7 \\
\hline $900(180,180,180,180,180)$ & 3467.4 & 3254.0 & 3143.3 & 3537.1 & 3474.0 & 3375.2 \\
\hline $1000(200,200,200,200,200)$ & 4458.6 & 4658.2 & 4987.6 & 5198.3 & 4819.3 & 4824.4 \\
\hline
\end{tabular}


Additionally, Tables III and IV illustrate the CPU utilization and the ASR values for the same DVE system (composed of 5 servers) when it is simulated under partitions where the term $C_{p}^{W}$ is maximized (the workload is unevenly distributed). Table III shows the performance results for a partition that minimizes the term $C_{p}^{L}$ (the number of inter-server messages), while the partition whose results are shown in Table IV maximizes this term. The most important result that Tables III and IV show is that the saturation point of a DVE system is reached when any of the servers reaches the $100 \%$ limit of CPU utilization. Effectively, in both tables only server 3 reaches $100 \%$ of CPU utilization for 650 avatars, and for that simulation ASR goes off in both tables. This effect cannot be clearly observed in Tables I and II because in those partitions the workload is perfectly balanced and, therefore, several servers reach $100 \%$ of CPU utilization in those tables simultaneously.

A line-by-line comparison of Tables III and IV show that the CPU utilization values are very similar in both tables. This result indicates that the term $C_{p}^{L}$ does not have any effect on the system throughput when the term $C_{p}^{W}$ is worsened (the system reaches saturation with 650 avatars in both tables). However, the system responses obtained for each server greatly differ between these two tables. When a given server reaches saturation (650 avatars, server S3), if the number of inter-server messages is minimized (Table III), then the avatars assigned to other servers do not use this server for communication purposes and, therefore, the saturation of server S3 does not affect the average latencies obtained for the rest of the servers. On the contrary, if the term $C_{p}^{L}$ is maximized (Table IV) then the saturated server is used by avatars assigned to other servers for sending updating messages. Therefore, the system responses for the rest of the servers are also greatly increased. This behavior explains the difference between these two tables in the ASR value obtained for 650 avatars. This value is 605 for 650 avatars in Table III, while it increases up to 1,623 in Table IV for the same number of avatars. That is, if the workload is not properly balanced then the saturation of a given server is propagated faster to other servers, greatly increasing the ASR.

A comparison of Tables III and IV with Tables I and II shows that balancing the workload (minimizing the term $C_{p}^{W}$ of quality function $C_{p}$ ) results in the improvement of the DVE system throughput (the number of avatars supported by the system while still providing a reasonable ASR). Effectively, the same DVE system composed of 5 servers reaches saturation with 800 avatars in Tables I and II, while it reaches saturation with 650 avatars in Tables III and IV. 
TABLE III

AVERAGE SYSTEM RESPONSES AND CPU UTILIZATION VALUES FOR A PARTITION WHERE THE TERM $C_{p}^{W}$ IS MAXIMIZED AND THE TERM $C_{p}^{L}$ IS MINIMIZED

\begin{tabular}{|c|c|c|c|c|c|c|}
\hline \multicolumn{7}{|c|}{ Utilization $(\%)$} \\
\hline Number of avatars & CPU1 & CPU2 & CPU3 & CPU4 & CPU5 & ASR \\
\hline $\mathbf{6 0 0}(120,120,120,120,120)$ & 55 & 53 & 50 & 56 & 51 & 237.2 \\
\hline $\mathbf{6 2 5}(120,120,145,120,120)$ & 59 & 50 & 74 & 58 & 53 & 246.0 \\
\hline $\mathbf{6 5 0}(120,120,170,120,120)$ & 52 & 51 & 100 & 54 & 54 & 605.9 \\
\hline $675(120,120,195,120,120)$ & 53 & 57 & 100 & 56 & 53 & 2874.6 \\
\hline $700(120,120,220,120,120)$ & 56 & 56 & 100 & 53 & 59 & 4824.4 \\
\hline \multicolumn{7}{|c|}{ System Responses (ms) } \\
\hline Number of avatars & SR-S1 & SR-S2 & SR-S3 & SR-S4 & SR-S5 & ASR \\
\hline $\mathbf{6 0 0}(120,120,120,120,120)$ & 234.4 & 243.8 & 231.3 & 253.9 & 222.8 & 237.2 \\
\hline $625(120,120,145,120,120)$ & 229.1 & 241.3 & 305.1 & 227.5 & 227.1 & 246.0 \\
\hline $\mathbf{6 5 0}(120,120,170,120,120)$ & 225.7 & 230.0 & 2101.8 & 232.0 & 239.9 & 605.9 \\
\hline $\mathbf{6 7 5}(120,120,195,120,120)$ & 237.0 & 241.3 & 13435.1 & 231.8 & 227.9 & 2874.6 \\
\hline $700(120,120,220,120,120)$ & 4458.6 & 4658.2 & 4987.6 & 5198.3 & 4819.3 & 4824.4 \\
\hline
\end{tabular}

However, there are no significant differences between the ASR values in the first two lines of table I and the corresponding values in table III, nor between the corresponding values of table II and table IV on the fi rst two lines. These results indicate that the term $C_{p}^{W}$ has an effect on the system throughput, but it does not have a signifi cant effect on the ASR. On the contrary, the term $C_{p}^{L}$ mainly affects the ASR values (system latency). However, this effect is signifi cant only when the system reaches saturation. If the system is working below the saturation point, then the impact of $C_{p}^{L}$ is not signifi cant. Therefore, this characterization study shows that the partitioning method should be targeted to balance the workload among the servers in the system, in such a way that none of them reaches $100 \%$ of CPU utilization. 
TABLE IV

Performance Results For a PARTITION Where both $C_{p}^{L}$ AND $C_{p}^{W}$ TERms ARE MAXIMized

\begin{tabular}{|c|c|c|c|c|c|c|}
\hline \multicolumn{7}{|c|}{ Utilization (\%) } \\
\hline Number of avatars & CPU1 & CPU2 & CPU3 & CPU4 & CPU5 & ASR \\
\hline $\mathbf{6 0 0}(120,120,120,120,120)$ & 57 & 58 & 51 & 57 & 50 & 244.4 \\
\hline $625(120,120,145,120,120)$ & 60 & 56 & 77 & 54 & 57 & 339.6 \\
\hline $\mathbf{6 5 0}(120,120,170,120,120)$ & 52 & 54 & 100 & 57 & 58 & 1623.4 \\
\hline $675(120,120,195,120,120)$ & 56 & 55 & 100 & 59 & 56 & 5087.0 \\
\hline $700(120,120,220,120,120)$ & 58 & 61 & 100 & 52 & 55 & 9825.9 \\
\hline \multicolumn{7}{|c|}{ System Responses (ms) } \\
\hline Number of avatars & SR-S1 & SR-S2 & SR-S3 & SR-S4 & SR-S5 & ASR \\
\hline $\mathbf{6 0 0}(120,120,120,120,120)$ & 241.4 & 239.6 & 241.0 & 261.5 & 238.7 & 244.4 \\
\hline $625(120,120,145,120,120)$ & 301.6 & 309.5 & 399.6 & 320.4 & 367.1 & 339.6 \\
\hline $\mathbf{6 5 0}(120,120,170,120,120)$ & 1012.5 & 1249.4 & 3129.5 & 1824.6 & 900.9 & 1623.4 \\
\hline $675(120,120,195,120,120)$ & 3996.4 & 4363.3 & 8313.4 & 4040.6 & 4721.1 & 5087.0 \\
\hline $700(120,120,220,120,120)$ & 1020.7 & 11212.3 & 21123.4 & 8556.4 & 7216.5 & 9825.9 \\
\hline
\end{tabular}

\section{A New Partitioning Method}

\section{A. Method Description}

The results in the previous section suggest that it is necessary to develop a partitioning method that is targeted to keep all the servers in the system below a certain threshold of CPU utilization, regardless of the amount of network traffi c.

Recently, a partitioning method (based on a load balancing technique) that takes into account the non-linear behavior of DVE servers has been proposed for solving the partitioning problem [4]. In this proposal, all objects within each region of the virtual world are managed by a given server, and an adaptive load balancing scheme is provided. Nevertheless, this scheme provides only local load balancing among the servers managing adjacent regions. The reason for such locality is the reduction of the number of inter-server messages (in this proposal, when the AOI of a given avatar crosses the boundary of two servers, both servers are responsible for transferring 
object models to that avatar). However, the local scope of the load balancing technique limits the performance of the partitioning method, since the workload supported by a given server can only be distributed among some of the servers (those managing adjacent regions), instead of using all servers. The performance evaluation of this proposal considers that all avatars in the virtual world move circularly, starting and ending at the same location. Under this simplifying assumption of a uniform movement pattern of avatars, local load balancing provides a good performance. However, it is very unlikely that all avatars in a DVE system move following such a uniform pattern. DVE systems usually have certain "hot-points" where avatars tend to head for [16]. In other cases, like in 3-D networked games, these hot-points are game resources (energy, weapons, etc.) that dynamically appear and disappear, and only those avatars located within a given radius of the hot-points tend to approach these points [30]. In these situations, all the neighboring regions around the hot points tend to get crowded with avatars, and therefore all the servers managing these regions tend to get saturated, while other servers support a low load. A global load balancing technique would use all the servers in the system, and it could provide better performance than a local load balancing technique for such cases.

We propose a partitioning method that (unlike the partitioning method proposed in [4]) performs an object-oriented management of avatars. Servers manage the workload generated by objects (avatars), rather than regions of the virtual world. This approach allows a global scope in the load balancing technique, using any server in the DVE system for load balancing purposes when a given server reaches saturation. Concretely, we propose the Avatar Load Balancing $(A L B)$ technique [36]. This proposal is based on a server-initiated load balancing technique developed for distributed operating systems [9]. ALB starts with an initial assignment of avatars to servers. In order to obtain this initial assignment, a GRASP-based algorithm [32] or any other algorithm can be used. As avatars move within the virtual world freely, both the CPU utilization and also the number of avatars that each server supports are constantly monitored. From these measurements, the workload that each avatar adds to its server is statistically computed, taking into account the two factors shown in [35]: the movement rate of that avatar and the presence factor $\left(P_{f}\right)$ of that avatar. In this way, the estimated workload that each avatar represents to its server is periodically estimated. When a given server $S_{x}$ reaches $99 \%$ of CPU utilization, then the ALB algorithm is started. The threshold value (99\%) should be tuned according to the system latency, in order to avoid that any server reaches $100 \%$ of CPU utilization during the 
time interval required to compute the ALB algorithm and to re-assign avatars. The purpose of the ALB algorithm is to select a number of avatars currently assigned to $S_{x}$ and to assign them to the server with the lowest CPU utilization (denoted as $S_{y}$ ), in such a way that the resulting estimated CPU utilization of $S_{x}$ is reduced to $90 \%$. The threshold value should be low enough to ensure that saturation is avoided in $S_{x}$, while, at the same time, it should be as high as possible in order to avoid the cascading effect (if all the servers in the system are close to saturation, $S_{y}$ may have a high CPU utilization. Assigning more avatars to $S_{y}$ may lead this server to reach saturation, which in turn leads to start the load balancing algorithm again).

The first step of the ALB algorithm consists of sorting the avatars currently assigned to $S_{x}$ by their presence factor $P_{f}$. Then, the fi rst avatars in this ranking are assigned to $S_{y}$, until the estimated workload of the re-assigned avatars represents at least $10 \%$ of CPU utilization in server $S_{x}$. The reason for using the criterion of the presence factor is that usually the avatars with the highest presence factor are also the closest avatars in the virtual world. Therefore, most of the messages generated by these re-assigned avatars will be intra-server messages. In this way, the proposed technique not only avoids the saturation of $S_{x}$ (the main goal of the load balancing technique) but it also tries to reduce the amount of inter-server messages (this feature helps to decrease system latency, as shown in the previous section).

\section{B. Evaluation Results}

In order to evaluate the performance of the ALB technique, we have evaluated the performance of different DVE configurations by simulation, as described in section III. For comparison purposes, we have simulated the proposed technique and the Adaptive Region Partitioning Technique (ARPS) [4], the only existing partitioning method that takes into account the non-linear behavior of DVE servers with the number of avatars assigned to them. We have simulated DVE systems with three different movement patterns of avatars: Changing Circular Pattern (CCP) [4], Hot-Points-ALL (HP-ALL) and also Hot-Point-Near (HP-Near). CCP considers that all avatars in the virtual world move circularly, starting and ending at the same location. HP-ALL considers that there are certain "hot-points" where all avatars tend to head for sooner or later, as shown in [16]. Finally, HP-Near also considers these hot-points; however, only those avatars located within a given radius of the hot-points tend to approach these points [30]. An iteration in a given movement pattern consists of all the avatars in the system performing a movement. A 
simulation consists of 100 iterations. Additionally, different initial distributions of avatars in the virtual world (uniform, skewed and clustered) have been considered, as in other studies [4], [27]. As an example, Figure 8 shows the fi nal distributions of avatars in a 2-D virtual world when the different movement patterns are applied and the initial distribution is a uniform distribution of avatars.
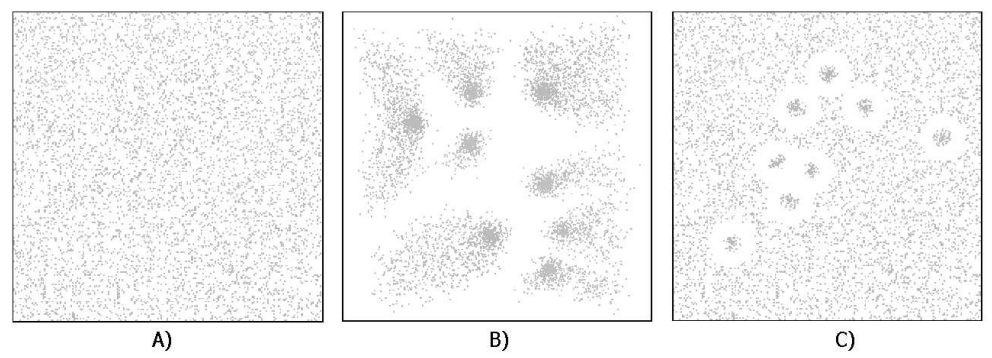

Fig. 8. Final distribution of avatars for a) CCP, b) HP-Near, and c) HP-All movement patterns applied to an initial uniform distribution of avatars

Although we have simulated all the movement patterns mentioned above in this section, we only present the results of the experiments performed with HP-Near and HP-ALL patterns. The reasons are both space limitations and also that the CCP movement pattern hardly starts the load balancing algorithm if the initial workload does not lead to system saturation, since all avatars follow a uniform circular path. The results for both a clustered and a skewed initial distribution are not presented either, since they only differ with the results presented here in the initial level of workload that the DVE system supports. In this section, we present the results for HP-ALL and HP-Near movement pattern of avatars and for a uniform initial distribution of avatars.

We have tested a large number of different DVE confi gurations, ranging from SMALL virtual worlds (composed of 3 servers and 180 avatars) to LARGE virtual worlds (composed of 6000 avatars and 9 servers). Due to space limitations, we only present the results for a LARGE DVE confi guration (the worst case for the partitioning method).

Tables V and VI show the performance evaluation results obtained for ARPS and ALB load balancing techniques when the initial distribution of avatars is the uniform distribution and avatars move following the HP-ALL pattern. Both tables show the percentage of CPU utilization in each server after different iterations of the simulation. Each row shows the CPU utilization for a different server, and each column shows the CPU utilization for all the servers and for a 
given partitioning method after a given iteration. Additionally, the last row shows the resulting sum of standard deviations $\sum \delta_{w}$ for the average estimated workload $w$ that DVE system servers support. This value measures how balanced the workload is after each iteration (the lower this value is, the better workload balancing is achieved). In order to make a fair comparison, in both strategies we have obtained the initial assignment of avatars by means of the same initial algorithm (the one proposed in [4]). Therefore, the fi rst column in Tables V and VI show the same CPU utilization. Since the HP-ALL pattern is applied to all avatars, all of them tend to head for a given location. As a consequence, the presence factor of avatars greatly increases and so does the total amount of workload. Therefore, the CPU utilization increases in all servers from the left to the right columns of the tables.

\section{TABLE V}

RESULTS FOR THE ARPS TECHNIQUE WITH AN INITIAL UNIFORM DISTRIBUTION OF AVATARS AND HP-ALL PATTERN

\begin{tabular}{|c|c|c|c|c|c|}
\hline & \multicolumn{5}{|c|}{ ARPS (Iteration number) } \\
& 1 & 10 & 11 & 12 & 13 \\
\hline S0 & 9.4 & 98.5 & 59.6 & 86.1 & 97.6 \\
\hline S1 & 9.4 & 10.6 & 10.7 & 11.2 & 15.3 \\
\hline S2 & 8.6 & 36.2 & 47.9 & 59.6 & 86.1 \\
\hline S3 & 9.8 & 64.7 & 88.4 & 96.7 & 100 \\
\hline S4 & 8.7 & 21.1 & 82.8 & 95.9 & 75.6 \\
\hline S5 & 11.6 & 48.9 & 67.4 & 83.3 & 81.9 \\
\hline S6 & 8.9 & 19.1 & 18.1 & 31.5 & 90.3 \\
\hline S7 & 9.3 & 57.7 & 75.9 & 99.2 & 100 \\
\hline S8 & 8.6 & 68.4 & 82.2 & 99.1 & 100 \\
\hline \hline$\sum \delta_{w}$ & 25 & 907 & 897 & 1069 & 997 \\
\hline
\end{tabular}

Close examination of Tables V and VI reveals that the DVE system quickly reaches saturation with the HP-ALL movement pattern, since only 14 out of 100 iterations could be simulated (Table VI, ALB technique) without reaching the saturation point. From that point, the overall workload generated by avatars is too big for this DVE confi guration (except servers $S 4$ and $S 6$, 
TABLE VI

RESUlts FOR THE ALB TECHNIQUE WITH AN INITIAL UNIFORM DISTRIBUTION OF AVATARS AND HP-ALL PATTERN

\begin{tabular}{|c|c|c|c|c|c|c|}
\hline & \multicolumn{6}{|c|}{ ALB (Iteration number) } \\
\hline S0 & 9.4 & 89.5 & 89.4 & 89.3 & 98.6 & 100 \\
\hline S1 & 9.4 & 42.1 & 52.7 & 88.5 & 97.2 & 100 \\
\hline S2 & 8.6 & 45.1 & 56.4 & 74.4 & 99.5 & 99.9 \\
\hline S3 & 9.8 & 75.0 & 93.8 & 89.4 & 97.4 & 100 \\
\hline S4 & 8.7 & 31.3 & 63.9 & 84.2 & 93.7 & 100 \\
\hline S5 & 11.6 & 55.6 & 69.5 & 78.5 & 98.3 & 99.6 \\
\hline S6 & 8.9 & 33.3 & 53.4 & 60.3 & 99.2 & 94.6 \\
\hline S7 & 9.3 & 74.2 & 92.8 & 89.5 & 96.6 & 98.4 \\
\hline S8 & 8.6 & 84.6 & 89.5 & 89.4 & 99.1 & 100 \\
\hline \hline$\sum \delta_{w}$ & 25 & 475 & 35 & 128 & 210 & 296 \\
\hline
\end{tabular}

all servers show a CPU utilization of $99 \%$ or more)

On the other hand, Tables V and VI show that the ALB method provides better balanced partitions than the ones provided by ARPS technique. As a result, the ARPS technique (Table V) only keeps the system below saturation until iteration 12. Indeed, servers $S 7, S 8$ and $S 3$ reach $100 \%$ of CPU utilization in the thirteenth iteration, while in this iteration server $S 1$ is below $16 \%$ of CPU utilization. In iteration 12 servers $S 7$ and $S 8$ reach $99 \%$ of CPU utilization, while server $S 1$ is below $12 \%$. The reason for this behavior is the local scope of the load balancing technique. Under this movement pattern, all avatars tend to continuously concentrate on some points or regions of the virtual world, and the servers managing these regions cannot share that workload with the servers managing remote regions (with few avatars). Thus, this partitioning method cannot avoid that servers $S 3, S 7$ and $S 8$ reach saturation in iteration 13. On the contrary, the ALB technique (Table VI) is able to share the workload among all the servers, and thus after iteration 11 the value of $\sum \delta_{w}$ is around half of the value provided by the ARPS method for the same iteration. As a result, the ALB technique keeps the system below the saturation 
point in iterations 12, 13 and 14, although in iteration 14 all servers are around $94 \%$ of $\mathrm{CPU}$ utilization. The system only reaches saturation (iteration 15) when the overall workload reaches the maximum workload that the system can support (as avatars concentrate, the number of avatars in the AOI of these avatars increases and, therefore, the workload that each avatar adds also increases).

Tables VII and VIII show the results for an initial uniform distribution of avatars and a HP-near movement pattern of avatars. Since in this pattern only some of the avatars tend to concentrate around the hot-points, the workload generated by avatars in this simulations is lower than the workload generated for the HP-ALL case, and it does not reach the total workload that the DVE system can support. Table VII shows that the ARPS technique can only keep the system below the saturation point until the 15th iteration. Again, servers managing the crowded regions reach $100 \%$ of CPU utilization, while servers managing distant regions are around $12 \%$ of CPU utilization. On the contrary, Table VII shows that the ALB technique balances the workload among all the servers, showing a signifi cantly lower value of $\sum \delta_{w}$ for the first 15 iterations. As a result, this technique is able to keep all servers below $100 \%$ of CPU utilization during the whole simulation (100 iterations).

\section{CONClusions AND Future Work}

In this paper, we have presented the experimental correlation of the quality function proposed in the literature for solving the partitioning problem $\left(C_{p}\right)$ with the performance of DVE systems. Since results show an absence of correlation, we have also proposed a characterization study of DVE systems. DVE systems show a non-linear behavior with the number of avatars in the system. The average system response (round trip-delay of messages notifying movements of avatars) remains practically invariant with the number of avatars in the system until the DVE system reaches a saturation point. This saturation point is given by the limit of $100 \% \mathrm{CPU}$ utilization in any of the servers. Since quality function $C_{p}$ does not take into account CPU utilization in order to measure the quality of a partition, it cannot take into account the nonlinear behavior of DVE systems with the number of avatars either.

We have also studied the effects of the two terms of $C_{p}$ (workload balancing and the amount of inter-server messages) on the performance of DVE systems. The results show that workload balancing mainly has an effect on system throughput, while the amount of inter-server messages 
TABLE VII

RESULTS FOR THE ARPS TECHNIQUE WITH AN INITIAL UNIFORM DISTRIBUTION OF AVATARS AND HP-NEAR PATTERN

\begin{tabular}{|c|c|c|c|c|c|c|}
\hline & \multicolumn{5}{|c||}{ ARPS (Iteration number) } \\
\hline S0 & 9.0 & 66.2 & 93.9 & 54.2 & 74.3 & 65.1 \\
\hline S1 & 9.1 & 9.0 & 8.8 & 8.5 & 8.5 & 12.7 \\
\hline S2 & 8.8 & 26.9 & 33.3 & 44.4 & 55.8 & 79.8 \\
\hline S3 & 9.8 & 50.6 & 64.4 & 88.7 & 97.2 & 59.6 \\
\hline S4 & 9.0 & 15.4 & 16.0 & 76.3 & 94.6 & 100.0 \\
\hline S5 & 11.3 & 35.5 & 42.4 & 60.0 & 74.2 & 100.0 \\
\hline S6 & 9.5 & 14.0 & 18.0 & 15.8 & 26.9 & 28.5 \\
\hline S7 & 9.2 & 37.5 & 53.0 & 69.5 & 91.0 & 100.0 \\
\hline S8 & 8.6 & 54.7 & 65.1 & 78.1 & 98.7 & 100.0 \\
\hline \hline$\sum \delta_{w}$ & 22 & 643 & 897 & 865 & 1030 & 1615 \\
\hline
\end{tabular}

mainly has an effect on system latency. However, if none of the servers reaches saturation, then the amount of inter-server messages does not have any significant effect on system latency. Therefore, in order to design an efficient and scalable DVE system, the partitioning method should be targeted to balance the workload among the servers in the system in such a way that none of them reaches $100 \%$ of CPU utilization.

Taking into account these results, we have proposed a partitioning method based on a load balancing technique. Unlike the only existing technique that takes into account the non-linear behavior of DVE servers with the number of avatars they support, in this approach servers manage the workload generated by avatars, not regions of the virtual world. The global scope of the proposed technique allows to balance the workload generated by all of the avatars as much as possible. We have evaluated the proposed technique by simulation. The results show that the proposed strategy can improve the DVE system performance, particularly for non-uniform movement patterns of avatars. Moreover, the proposed strategy allows to keep the DVE system below the saturation level if the overall workload generated by all of the avatars is lower than 
TABLE VIII

RESUlts FOR THE ALB TECHNIQUE WITH AN INITIAL UNIFORM DISTRIBUTION OF AVATARS AND HP-NEAR PATTERN

\begin{tabular}{|c|c|c|c|c|c|c|c|c|}
\hline & \multicolumn{7}{|c|}{ ALB (Iteration number) } \\
\hline S0 & 9.0 & 66.2 & 93.9 & 89.4 & 98.4 & 97.7 & 98.4 & 91.6 \\
\hline S1 & 9.1 & 9.0 & 8.8 & 38.9 & 89.5 & 96.5 & 87.5 & 92.6 \\
\hline S2 & 8.8 & 26.9 & 33.3 & 45.7 & 87.6 & 95.4 & 97.5 & 89.9 \\
\hline S3 & 9.8 & 50.6 & 64.4 & 81.1 & 74.1 & 89.6 & 89.6 & 98.6 \\
\hline S4 & 9.0 & 15.4 & 16.0 & 53.6 & 65.2 & 88.5 & 90.0 & 97.5 \\
\hline S5 & 11.3 & 35.5 & 42.4 & 52.9 & 89.6 & 94.6 & 90.4 & 90.2 \\
\hline S6 & 9.5 & 14.0 & 18.0 & 22.5 & 95.2 & 97.6 & 97.5 & 97.2 \\
\hline S7 & 9.2 & 37.5 & 53.0 & 65.9 & 96.6 & 96.9 & 94.1 & 94.3 \\
\hline S8 & 8.6 & 54.7 & 65.1 & 79.6 & 97.1 & 98.1 & 77.5 & 92.9 \\
\hline \hline$\sum \delta_{w}$ & 22 & 643 & 897 & 214 & 74 & 89 & 61 & 40 \\
\hline \hline
\end{tabular}

the total workload that the DVE system can support, regardless of both the movement pattern and the initial distribution of avatars in the virtual world.

\section{REFERENCES}

[1] H. Abrams, K. Watsen and M. Zyda, "Three Tiered Interest Management for Large-Scale Virtual Environments", in Proc. of ACM VRST 98, November 1998, Taipei.

[2] M. Abrash, "Quakeś game engine: The big picture”, in Dr. Dobb’s Journal, Spring 1997.

[3] D.B.Anderson, J.W.Barrus, J.H.Howard, "Building multi-user interactive multimedia environments at MERL", in IEEE Multimedia, 2(4), pp.77-82, Winter 1995.

[4] N.Beatrice, S.Antonio, L.Rynson and L.Frederick, "A Multi-Server Architecture for Distributed Virtual Walkthrough", in Proc. of ACM VRST 02, pp. 163-170, Nov. 2002.

[5] C. Bouras, D. Fotakis, A. Philopoulos, "A Distributed Virtual Learning Centre in Cyberspace", Proc. of the 4th International Conference on Virtual Systems and Multimedia, VSMM98, Gifu-Japan, 18-20 November 1998

[6] P.A.Berstein, V.Hadzilacos and N.Goodman, "Concurrency, Control and Recovery in Database Systems", AddisonWesley. 1997.

[7] T. Carneiro and J. Árabe, "Load Balancing for Distributed Virtual Reality Systems", In Proceedings of International Symposium on Computer Graphics, Image Processing, and Vision (SIBGRAPI'98), pp. 158-165, January 1998 
[8] M. Capps, "The QUICK Framework for Task-Specifi c Asset Prioritization in Distributed Virtual Environments", In proceedings of IEEE Virtual Reality 2000, East Rutherford, NJ, March 2000.

[9] R.Chow and T.Jhonson, "Distributed Operating Systems \& Algorithms", Addison Wesley-Longman, 1997.

[10] DIS. 1278.1 IEEE Standard for Distributed Interactive Simulation-Application Protocols (ANSI). DMSO. DoD High Level Architecture. 1997.

[11] J. Falby, M. Zyda, D. Pratt, and R. Mackey. "NPSNET: Hierarchical Data Structures for Real-Time ThreeDimensional Visual Simulation”. Computers \& Graphics, 17(1):65-69, 1993.

[12] FIPA Agent Management Specification. Foundation for Intelligent Physical Agents, 2000. Available at http://www.fipa.org/specs/fipa00023/

[13] T.A. Funkhouser, "Network Topologies for Scalable Multi-User Virtual Environments", IEEE VRAIS '96, San Jose, CA, April, 1996.

[14] E. Frecon, M. Stenius, "DIVE: A scalable network architecture for distributed virtual environments", Distributed Systems Eng., Vol. 5, 1998.

[15] F.C. Greenhalgh "Awareness-based communication management in MASSIVE systems", in Distributed Systems Engineering, Vol. 5, 1998.

[16] C. Greenhalgh, "Analysing Movement and World Transitions in virtual Reality Tele-conferencing", in 5th European Conference on Computer Supported Cooperative Work (ECSCW'97), Lancaster, U.K., September 1997.

[17] C. Greenhalgh, Adrian Bullock, Emmanuel Frécon, David Llyod and Anthony Steed, "Making Networked Virtual Environments Work ”, Presence, Vol. 10, Issue 2, April 2001.

[18] E.P. Harvey, R.L. Schaffer, S.M. McGarry "High performance fi xed-wing aircraft simulation using SIMNET protocols", Proc. of 23th. Annual Summer Computer Simulation Conference. Baltimore, USA. pp. 965-970. SCS. 22-24 July 1991.

[19] J.C.Hu, I.Pyarali, D.C.Schmidt, "Measuring the Impact of Event Dispatching and Concurrency Models on Web Server Performance Over High-Speed Networks", Proc. of the 2nd. IEEE Global Internet Conference, November.1997.

[20] Kuhl, F., Weatherly, R, Dahmann, J., "Creating Computer Simulation Systems: An Introduction to the High Level Architecture", Prentice-Hall PTR, Upper Saddle River, NJ, 1999.

[21] Y. Kawahara, H. Morikawa and T. Aoyama, "A Peer-to-Peer Message Exchange Scheme for Large Scale Networked Virtual Environments", Proc. of 8th IEEE Int. Conf. on Communications Systems (ICCS2002), November 2002.

[22] D. Lee, M. Lim, and S. Han, "ATLAS - A Scalable Network Framework for Distributed Virtual Environments", ACM Collaborative Virtual Environments(CVE2002), Bonn Germany, September-October, 2002, pp.47-54.

[23] Michael Lewis and Jeffrey Jacboson, "Game Engines in Scientifi c Research", in Communications of the ACM, Vol 45. No.1, January 2002.

[24] John C.S. Lui, M.F.Chan, Oldfi eld K.Y, "Dynamic Partitioning for a Distributed Virtual Environment", Department of Computer Science, Chinese University of Hong Kong, 1998.

[25] John C.S. Lui, W.K. Lam, "General Methodology in Analysing the Performance of Parallel/Distributed Simulation under General Computational Graphs", in Third International Conference on the numerical Solution of Markov Chain, September 6-10, 1999.

[26] John C.S. Lui, M.F. Chan, K.Y. So, T.S. Tam. "Deriving An Optimal Synchronization Interval for Supporting View Consistency in a Distributed Virtual Environment", in Fifth International Workshop on Multimedia Information Systems (MIS’99), pp 99-106, October 1999. 
[27] Jonh C.S. Lui, M.F. Chan, "An Effi cient Partitioning Algorithm for Distributed Virtual Environment Systems”, IEEE Trans. Parallel and Distributed Systems, Vol. 13, March 2002

[28] Michael R. Macedonia, "A Taxonomy for Networked Virtual Environments", in IEEE Multimedia, Vol. 4 No. 1, pp 48-56. January-March, 1997.

[29] Macedonia, Michael R., Zyda, Michael J., Pratt, David R., Brutzman, Donald P., Barham P. T. ”Exploiting Reality with Multicast Groups,” IEEE Computer Graphics \& Applications, September 1995, pp.38-45.

[30] M. Matijasevic, K. P. Valavanis, D. Gracanin, I. Lovrek, "Application of a Multi-User Distributed Virtual Environment Framework to Mobile Robot Teleoperation over the Internet”, in Machine Intelligence \& Robotic Control, Vol. 1, No. 1, pp. 11-26 (1999).

[31] D.C.Miller, J.A. Thorpe, "SIMNET: The advent of simulator networking", in Proceedings of the IEEE, 83(8), pp. 1114-1123. August, 1995.

[32] P. Morillo, M. Fernández, "A GRASP-based Algorithm for Solving DVE Partitioning Problem", Proc. of 2003 Int. Parallel and Distributed Processing Symposium (IPDPS' 2003), Nice, France. April, 2003.

[33] P. Morillo, M Fernández, J.M. Orduña, "A Comparison Study of Moder Heuristics for Solving the Partitioning Problem in Distributed Virtual Environment Systems", in Intl. Conf. on Computational Science and its Applications (ICCSA’ 2003), pp. 458-467. May 2003. Lecture Notes in Computer Science 2669. Springer-Verlag.

[34] P. Morillo, J.M. Orduña, M. Fernández, J. Duato, "On the Characterization of Distributed Virtual Environment Systems”, in European Conference on Parallel Processing (Euro-Par' 2003), pp. 1190-1198. August, 2003. Lecture Notes in Computer Science 2790. Springer-Verlag.

[35] P. Morillo, M. Fernández, J. M. Orduña, "On the Characterization of Avatars in Distributed Virtual Worlds", in EUROGRAPHICS' 2003, pp. 215-220 (short presentations), September 2003.

[36] P. Morillo, J.M. Orduña, M. Fernández and J. Duato, “An Adaptive Load Balancing Technique for Distributed Virtual Environment Systems", Proceedings of the 15th IASTED International PDCS-03, California, USA. November, 2003.

[37] J.M.Salles Dias, Ricardo Galli, A. C. Almeida et al, "mWorld: A Multiuser 3D Virtual Environment", in IEEE Computer Graphics, Vol. 17, No. 2, March-April 1997.

[38] J. Smed, T. Kaukoranta and H. Hakonen, “ A Review on Networking and Multiplayer Computer Games”, Tech Report 454, Turku Centre for Computer Science, April 2002

[39] S.Singhal, and M.Zyda, "Networked Virtual Environments. Design and Implementation”. Addison-Wesley, July 1999.

[40] P.T.Tam, "Communication Cost Optimization and Analysis in Distributed Virtual Environment", M. Phil second term paper, Technical report RM1026-TR98-0412. Department of Computer Science \& Engineering.The Chinese University of Hong Kong. 1998.

[41] H. Trefftz, I. Marsic, and Michael Zyda, "Handling Heterogeneity in Networked Virtual Environments", Presence, Vol. 12, No. 1, pp.38-52, February 2003.

[42] L. Zou, M. Ammar, C. Diot, "An Evaluation of Grouping Techniques for State Dissemination in Networked MultiUser Games", in Proceedings of the Ninth International Symposium on Modelling, Analysis and Simulation of Computer and Telecommunication Systems, (MASCOTS'01), August 2001. 